\title{
Toxoplasma gondii infections among pregnant women, children and HIV- seropositive persons in Accra, Ghana
}

Irene Ayi ${ }^{1 *}$, Augustine Odoi-Kpoti Sowah ${ }^{2,4}$, Emmanuel Awusah Blay ${ }^{1,3}$, Takashi Suzuki $^{3}$, Nobuo Ohta ${ }^{3}$ and Patrick F. Ayeh-Kumi ${ }^{4}$

\begin{abstract}
Background: Toxoplasma gondii infection can lead to severe disease outcomes in immune-compromised people. This study sought to determine the seroprevalence of anti-T. gondii antibodies among pregnant women, hospitalized children ( $<5$ years old) and HIV-seropositive persons in Accra.

Methods: A cross-sectional study was conducted in two hospitals in Accra, and a total of 450 voluntary participants were recruited for the study consisting of 125 pregnant women, 200 children and $125 \mathrm{HIV}$-seropositive persons. Serum was obtained from venous blood safely drawn from each participant and tested for specific anti-Toxoplasma antibodies IgG and IgM by ELISA. A serological criterion for seropositivity was a positive test result for any of the two anti-Toxoplasma antibodies or a combination of both. Questionnaire interviews were conducted to obtain personal information and Toxoplasma infection risk-related data.

Results: Those who tested seropositive for anti-T. gondii antibodies were $51.2 \%$ (64/125) pregnant women, $58.0 \%$ $(116 / 200)$ children and $57.6 \%$ (72/125) HIV patients. The major risk factors associated with anti-T. gondii seropositivity were identified as age (in children), handling raw meat and gravida status (in pregnant women). The results of this study confirmed that the seroprevalence of T. gondii infection is high among pregnant women, hospitalized children $<5$ years old and HIV patients.

Conclusions: A further study to investigate pre-pregnancy infections with T. gondii among women of childbearing age, seroconversion rate in pregnant women, rate of mother-to-child transmission and reactivated infections among HIV-seropositive persons is recommended.
\end{abstract}

Keywords: Toxoplasma gondii, Anti-Toxoplasma gondii antibodies, Immune-compromised

\section{Background}

Toxoplasmosis is one of the world's most common parasitic infections caused by Toxoplasma gondii, a ubiquitous intracellular protozoan parasite of warm-blooded animals [1-3]. The parasite is known to infect most genera of warm-blooded animals, including humans, but the definitive host is the felids (cat). Toxoplasmosis is a self-limiting disease with the parasite encysting after few weeks of infection, but reactivation of infection may occur even in

\footnotetext{
* Correspondence: iayi@noguchi.ug.edu.gh

'Department of Parasitology, Noguchi Memorial Institute for Medical

Research, College of Health Science, University of Ghana, P. O. Box LG 581,

Legon, Accra, Ghana

Full list of author information is available at the end of the article
}

healthy individuals due to several factors including suppression of one's immune system leading to significant morbidity and mortality $[4,5]$.

Seroepidemiological surveys have revealed varying degree of prevalence in terms of different geographical settings and the risk factors for acquiring the disease [6]. There have also been reports of varying prevalence in terms of age distribution, climate and socio-economic status [7-9]. Persons at risk of acquiring toxoplasmosis as an opportunistic disease usually are immune-compromised or immune-suppressed persons [10] due to the ability of the parasite to be reactivated from encysted bradyzoites to circulating tachyzoites [11]. 
In most European countries such as France and Austria, there are instituted national programmes that aim to screen pregnant women for toxoplasmosis, and such interventions have helped reduce the rate of congenital transmission and the rate of seroconversion due to early diagnosis $[12,13]$. During pregnancy, there is the occurrence of a high cascade of complex physiological conditions that enables the mother to fully adapt to the environment. Immune-suppression is noted to be a physiological change that the body of the mother induces for the prevention of foetal rejection as the foetus is considered as an allograft. This, therefore, makes the pregnant woman susceptible to many opportunistic infections [14]. However, in Ghana, like most sub-Saharan African countries, there is no national programme for screening pregnant women for toxoplasmosis.

HIV infections on the other hand cause progressive depletion of the $\mathrm{CD}^{+}{ }^{+} \mathrm{T}$ cells, which leads to lifethreatening opportunistic infections (OIs) or malignancies during the natural course of the disease [15]. Thus, in chronic toxoplasmosis infected patients, there can be reactivation of a latent infection which can cause lifethreatening encephalitis [16].

Primary infection of $T$. gondii during pregnancy can result in the vertical transmission of tachyzoites which can have severe consequences on the foetus such as retinochoroiditis, hydrocephalus and intracranial calcification of the children and long-term ocular lesions [17].

In Ghana, however, until recently when attention is being given to toxoplasmosis [18, 19], there is not much evidence-based information on T. gondii infections among high-risk groups in the populace. This therefore makes it difficult for the formulation and adoption of a national policy for toxoplasmosis management. We therefore sought to determine the level of exposure of infection among HIV-seropositive people, pregnant women and hospitalized children to T. gondii due to their suppressed or underdeveloped immune status.

\section{Methods}

\section{Study area}

The ante-natal unit, Obstetrics and Gynaecology Department and the Fevers Unit, Medical Department both at the Korle-Bu Teaching Hospital (KBTH) served as the recruiting sites for pregnant women and HIV-seropositive patients, respectively. KBTH is the leading tertiary referral hospital in Ghana with over 2000 bed capacity. The Princess Marie Louise Children's Hospital (PMLCH), an exclusively referral children's hospital in Accra, served as the site for recruiting children $<5$ years old.

\section{Study population}

The study population consisted of pregnant women aged $>18$ years (in any trimester of pregnancy) attending ante-natal clinic, male and female Outpatient Department attendees of the Fevers Unit, aged above 18 years who were HIV-seropositive and not yet started treatment with antiretroviral therapy and all at the KBTH as well as children of both sexes aged $<5$ years admitted at the PMLCH.

\section{Sampling and sample size}

For calculation of the sample size, we assumed a minimum of $10 \%$ and a maximum $90 \% T$. gondii seroprevalence as expected frequency of the factor under study and a confidence level of $95 \%$. The result of the calculation was a minimum of 138 subjects for accurate statistical inference [20]. A convenience sampling method was used to sample for the participants, and elements of bias were controlled by ensuring that the sample was a miniature of the whole population under study.

\section{Sample collection}

For participants aged above 18 years, 3-5 $\mathrm{ml}$ of blood was drawn from the cubital vein using a sterile hypodermic syringe, and the blood was transferred into labeled serum separator tubes. For children $<5$ years old, $3 \mathrm{ml}$ of blood was obtained from heel prick and transferred into labeled serum separator tubes. All blood samples were transferred in cool boxes containing ice-packs to the laboratories of the Department of Parasitology, Noguchi Memorial Institute for Medical Research (NMIMR), for further analysis. Serum was obtained from each blood sample by centrifugation at $14,000 \mathrm{rpm}$ for $10 \mathrm{~min}$ and stored at $-20{ }^{\circ} \mathrm{C}$ until assayed for anti- $T$. gondii antibodies.

\section{Socio-demographic and toxoplasmosis risk-related data collection}

Close-ended questionnaires were administered to collect demographic as well as toxoplasmosis-related risk factors data. Questionnaires were administered in an interview format in participants' mother language for easy understanding.

Questions sought personal information on participants including their knowledge about the disease and exposure to possible Toxoplasma infection transmission risk factors. The possible infection transmission risk factors considered in the questionnaire included handling and eating of meat from high transmission risk animals (pigs, sheep and goats) and contact with cat. With reference to meat, they were asked how often they handled and/or ate the meat and what form they preferred their meat (thoroughly and/or partially cooked). The pregnant women and the parents of the children were also asked if they owned cats or pets or had cats in their house or immediate surroundings. 


\section{Anti-T. gondii lgG and IgM detection by enzyme-linked} immunosorbent assay

Each sample was tested for the presence of anti- T. gondii antibodies, IgG and IgM, using commercial ELISA kits (CTK Biotech. Inc., San Diego, CA, USA) and following manufacturer instructions. Briefly, $100 \mu \mathrm{l}$ (for IgG) and $50 \mu \mathrm{l}$ (for IgM) of sample diluent was added to the test wells of the 96-well microtitre plates (pre-coated with $T$. gondii antigen), and $10 \mu \mathrm{l}$ of each test serum was added to each test well. The wells were then incubated at $37^{\circ} \mathrm{C}$ for 30 min after which they were completely and thoroughly washed with wash buffer. One hundred microlitres of HRP-anti-human IgG conjugates or HRP-anti-human IgM conjugates (depending on the test) were added to each of the wells except the blank wells and incubated at $37^{\circ} \mathrm{C}$ for $20 \mathrm{~min}$ after which wells were thoroughly washed. Fifty microlitres each of $\operatorname{TMB}(3,3$ ', 5, 5'-tetramethylbenzidine) substrates $A$ and $B$ was added to the test wells. The 96-well microplate was then incubated in the dark for $10 \mathrm{~min}$ at $37^{\circ} \mathrm{C}$, and the reaction was stopped by adding $50 \mu$ of stop buffer. Optical densities (OD) were then measured using a 450-nm ELISA plate reader, Multiskan ${ }^{\text {тм }}$ Microplate Absorbance Plate Reader (Thermo Scientific, USA). The cut-off value was given as $N+0.15$, where $N$ was the mean OD of the negative control. The OD of each specimen was calculated as the ratio of the specimen OD and the cut-off value. The mean OD value of the $T$ gondii IgG and IgM positive controls was expected to be $\geq 1.0$ and that of negative controls was expected to be $\leq 0.10$. Samples with an OD ratio $<1.00$ was considered to be negative with samples having an OD ratio $\geq 1.00$ considered positive.

\section{Statistical analysis}

Data analysis was performed using SPSS version 16.0. Difference in non-scalable variables such as age and sex of the study populations were assessed by Mann-Whitney $U$ rank sum with $p$ values $<0.05$ considered significant (CI $95 \%$ ). Chi-square $\left(\chi^{2}\right)$ test was used to determine associations between categorical variables. $p$ values $<0.05$ was considered statistically significant. Univariate analysis was used to predict the association between $T$. gondii seropositivity and risk factors.

\section{Results}

\section{Participant characteristics}

A total of four hundred and fifty (450) patients participated in this study. They comprised of 125 pregnant women aged 16 to 44 years (mean $28.25 \pm 3.65$ ) across trimesters; 200 children aged 0 to 5 years (mean $2.62 \pm 1.31$ ); and $125 \mathrm{HIV}$-seropositive persons aged 15 to 68 years (mean $43.24 \pm 4.97$ ) (Table 1 ). There was a significant difference between the ages of the study groups.

\section{Prevalence of anti-T. gondii IgG and IgM antibodies}

Seroprevalence of anti $-T$. gondii IgG among pregnant women, children and HIV-seropositive persons were $51.2 \%(64 / 125), 58.0 \%(116 / 200)$ and $57.6 \%(72 / 125)$, respectively. A seroprevalence of $0.5 \%(1 / 200)$ anti- $T$. gondii IgM was recorded among the children. However, there was no record of anti-T. gondii IgM in pregnant women and HIV-seropositive persons (Table 2).

Test of association between anti-T. gondii seropositivity and socio-demographic variables in pregnant women and HIV-seropositive persons

Demographic and clinical factors were related to seropositivity of anti-T. gondii antibodies. Results indicated no significant association between gender and the seropositivity for anti-T. gondii IgG. Furthermore, factors such as age (all study participants), stage of pregnancy and $\mathrm{CD}^{+} \mathrm{T}$ cell count $/ \mathrm{mm}^{3}$ were found not to have any statistical significance among pregnant women and HIVseropositive persons, respectively. However, there was a significant association between the gravida status of the pregnant women and anti-T. gondii seropositivity (Table 3). Exposure to infection risk factors such as owning a cat and contact with soil (due to their daily activities) as well as the handling and eating of raw or undercooked meat did not show any statistically significant associations with anti-T. gondii seropositivity in both pregnant women and HIV-seropositive persons. However, there was a significant association between handling of meat (beef, pork, mutton and chevron; among pregnant women only) and anti- $T$. gondii IgG positivity ( $p=0.02$; CI $95 \%$ ) (Table 4 ).

Table 1 General characteristics of study participants

\begin{tabular}{lllll}
\hline & & Study participants & & \\
\cline { 3 - 4 } & & Pregnant women & Children $<5$ years old & HIV-seropositive \\
& Number sampled & 125 & 200 & 125 \\
\hline Age (years) & Range & $16-44$ & $0-5$ & $15-68$ \\
& Mean & $28.25 \pm 3.65$ & $2.62 \pm 1.31$ & $43.24 \pm 4.97$ \\
Gender & Male (\%) & - & $109(54.5)$ & $54(43.2)$ \\
& Female (\%) & $125(100)$ & $91(45.5)$ & $71(56.8)$ \\
\hline
\end{tabular}


Table 2 Seroprevalence of anti-Toxoplasma gondii antibodies among study participants

\begin{tabular}{llll}
\hline T. gondii seropositivity $n(\%)$ & & \\
\hline Study participants & Number tested & IgG only & Both IgG and IgM \\
\hline Pregnant women & 125 & $64(51.2)$ & 0 \\
Children & 200 & $116(58.0)$ & $1(0.5)$ \\
HIV-seropositive & 125 & $72(57.6)$ & 0 \\
\hline
\end{tabular}

\section{Prevalence of anti-T. gondii antibodies in children}

Children in all age groups tested for anti- $T$. gondii antibodies showed a significant association with age and seropositivity with $p$ values $<0.05$ (CI $95 \%$ ). Results indicated an apparent linear increase in seropositives in the different ages of the children as shown in the Table 3. The lowest seroprevalence of $22.2 \%(6 / 27)$ was recorded in the 0 - to 0.5 -year-old group and is likely to be circulating maternal antibodies and steadily increased in the other age groups with the highest seroprevalence of $71.6 \%(53 / 74)$ being recorded in children aged between 3 and 5 years.

\section{Discussion}

The current study was conducted to determine the seroprevalence of anti- $T$. gondii antibodies among immunesuppressed groups of people represented by pregnant women, children and HIV-seropositive persons in Accra. All three groups recorded serum anti-T. gondii antibodies positivity of more than $50 \%$. A previous study among pregnant women (mean age of 28.1 years) in Accra, Ghana, yielded a seroprevalence of $92.5 \%$ [18]. However, another hospital-based study in patients of mean age 30.2 years visiting the Korle-Bu Teaching Hospital in Accra reported a lower prevalence rate of $49.7 \%$ [19]. A recent population-based study in coastal Ghana also reported a seroprevalence of $85 \%$ [8]. These disparities in toxoplasmosis seroprevalence estimates could be due to several factors such as study and geographical and climatic factors which are known to influence seroepidemiological studies [21]. Similar studies in other African countries have revealed varying prevalence such as $58.4 \%$ in Tunisia [22], 53.6 \% in Benin [23] and 57.9 \% in Egypt [24].

This study showed no significant association between anti- $T$. gondii antibody detection and presence of domestic cats, and this is in contrast with the results of studies reported in Nigeria [25], India [26] and Tanzania [27]. As IgM is considered as an indicator for recent/acute infection, our data suggests that there were no recent active infections in the pregnant women nor HIV-seropositive persons. The non-detection of IgM suggests that there were no recent infections; however, the presence of IgG could be due to reactivated or past infections [28].

It is crucial in pre- natal screening to ascertain whether $T$. gondii infection was acquired before or after conception
$[12,14]$. Primary congenital toxoplasmosis can lead to parasite transmission to the foetus via the placenta where the risk of transmission increases during gestation [29]. In seroepidemiological studies for $T$. gondii infection, the detection of anti-IgM antibodies in combination with antiIgG antibodies is always an indication of acute infections [30]. The reason is that IgM antibodies are not usually in acquired immunity and very rare in chronic infections. Furthermore, the presence of IgG together with IgM in circulation indicates recent acquired infection because; IgM antibodies wane down rapidly following recently acquired infections [18].

The current study recorded an overall seroprevalence of anti- $T$. gondii infection in children to be $58.5 \%$ for anti-T. gondii IgM and/or anti-T. gondii IgG. One (1) male infant aged 4 years tested positive for anti- $T$. gondii IgM. This is an indication of primary infection. Considering his age infection, he must have acquired the infection through exposure to any of the potential related risk factors. The seroprevalence recorded in this study was higher than the values reported for children of similar ages in a Nigerian study which reported $23.8 \%$ seroprevalence [31]. The difference in seropositivity rates could probably be due to the diagnostic techniques used which has variable sensitivities and specificities and also due to the local potential relative risk factors associated with the geographical areas.

This study found a statistically significant association between seroprevalence and age in children. Findings from the study showed a linear increase from the $<6$ months old children which recorded a $22.2 \%$ seroprevalence to the 3 - to 5-year-old children which recorded a seroprevalence of $71.6 \%$. This observation was in agreement with studies done elsewhere, in which the seroprevalence of T. gondii infection was found to increase with age [10, 32]. Previous studies had observed that there was a rapid increase in anti-T. gondii IgG seroprevalence from ages 6 months to 5 years and then plateaued through age 10 years [33]. One hypothesis is that this trend simply reflects the increasing number of "exposure years" as children aged [33]. As children tend to grow, they get in contact with soil which is known to be a high-risk factor in terms of exposure to $T$. gondii oocysts. Also in children <6-month-old age group, the IgG antibodies found are likely to be circulating maternal antibodies which has been passively passed on to the children or may be due to congenital infection. Establishment of congenital transmission however depends on the detection of parasite DNA in the foeto-placental region of the placenta $[1,28,34]$, and thus, anti-T. gondii antibodies will not be a conclusive diagnostic test.

Although pre-pregnancy $T$. gondii infection status of the pregnant women is unknown, it could be deduced from the antibodies detected and age of pregnancy that there is a possibility of having acquired the infection 
Table 3 Demographics associated with Toxoplasma seropositivity in participants by univariate analysis

\begin{tabular}{|c|c|c|c|c|}
\hline Study participants & Number examined $(N)$ & Anti-Toxoplasma gondii seropositivity $(n / N ; \%)$ & $x^{2}$ value & $p$ value $(\mathrm{Cl} 95 \%)$ \\
\hline Pregnant women & 125 & $64(51.2)$ & & \\
\hline \multicolumn{5}{|l|}{ Age groups } \\
\hline $16-20$ & 4 & $4(100)$ & 3.94 & $<0.05^{*}$ \\
\hline $21-30$ & 57 & $30(52.6)$ & 0.08 & 0.76 \\
\hline $31-40$ & 58 & $27(46.6)$ & 0.94 & 0.33 \\
\hline $41-44$ & 6 & $3(50.0)$ & 0.04 & 0.95 \\
\hline \multicolumn{5}{|l|}{ Stage of pregnancy } \\
\hline 1st trimester & 7 & $4(57.1)$ & 0.11 & 0.74 \\
\hline 2nd trimester & 38 & $22(57.8)$ & 0.98 & 0.32 \\
\hline 3rd trimester & 80 & $38(47.5)$ & 1.21 & 0.26 \\
\hline \multicolumn{5}{|l|}{ Gravida status of women } \\
\hline Primigravids & 49 & $17(34.7)$ & 8.79 & $0.003^{*}$ \\
\hline Multigravids & 76 & $47(61.8)$ & & \\
\hline HIV-seropositive persons & 125 & $72(57.6)$ & & \\
\hline \multicolumn{5}{|l|}{ Age groups } \\
\hline $15-24$ & 4 & $1(25.0)$ & 1.79 & 0.17 \\
\hline $25-34$ & 27 & $16(59.3)$ & 0.04 & 0.84 \\
\hline $35-44$ & 51 & $26(50.9)$ & 1.54 & 0.21 \\
\hline $45-54$ & 30 & $20(66.7)$ & 1.32 & 0.25 \\
\hline $55-64$ & 12 & $8(66.7)$ & 0.44 & 0.50 \\
\hline $65-68$ & 1 & $1(100)$ & 0.74 & 0.389 \\
\hline \multicolumn{5}{|l|}{ Gender } \\
\hline Male & 54 & $32(59.2)$ & 0.10 & 0.74 \\
\hline Female & 71 & $40(56.3)$ & & \\
\hline \multicolumn{5}{|c|}{$\mathrm{CD}^{+} \mathrm{T}$ cell count cell $/ \mathrm{mm}^{3}$} \\
\hline$<200$ & 9 & $6(66.7)$ & 0.32 & 0.56 \\
\hline $200 \geq \mathrm{CD}^{+} \leq 500$ & 64 & $38(59.4)$ & 0.16 & 0.68 \\
\hline$>500$ & 52 & $28(53.8)$ & 0.52 & 0.47 \\
\hline Children $<5$ years of age & 200 & $117(58.5)$ & & \\
\hline \multicolumn{5}{|l|}{ Age groups } \\
\hline $0-6$ months & 27 & $6(22.2)$ & 26.46 & $<0.001^{*}$ \\
\hline 6-12 months & 30 & $9(30.0)$ & 11.80 & $<0.001 *$ \\
\hline $1-3$ years & 69 & $48(69.6)$ & 5.31 & $0.02^{*}$ \\
\hline 3 years $>x<5$ years & 74 & $53(71.6)$ & 7.32 & $<0.001 *$ \\
\hline \multicolumn{5}{|l|}{ Gender } \\
\hline Male & 109 & $68(62.4)$ & 1.18 & 0.27 \\
\hline Female & 91 & 49 (53.8) & & \\
\hline
\end{tabular}

$p$ value was calculated by Pearson's chi-square $\left(x^{2}\right)$ with confidence interval $(\mathrm{Cl})$ of $95 \%$ and 1 degree of freedom ( $\left.d f\right)$. $p$ values less than 0.05 were considered statistically significant and are indicated in bold fonts $\left({ }^{*}\right)$

before conception or during pregnancy. Previous studies have shown that $T$. gondii infections acquired by the mother during the first trimester of gestation has a $10-15 \%$ risk of being transmitted congenitally to the foetus with severe consequences [14] whereas mothers contracting the infection during the second or third trimesters has an increasing (up to $68 \%$ ) risk of infecting their unborn babies, with less severe consequences [14]. This is therefore an indication that almost all the seropositive pregnant women identified in this study stand a risk of transmitting the infection to their foetuses. However, this study cannot conclusively ascertain whether there 
Table 4 Association of infection risk variables with Toxoplasma gondii seropositivity in pregnant women and HIV patients by univariate analysis

\begin{tabular}{|c|c|c|c|c|c|}
\hline Study participants & Factor & $\begin{array}{l}\text { Number examined, } N \\
\left(N / N_{0} ; \%\right)\end{array}$ & $\begin{array}{l}\text { Anti-Toxoplasma gondii seropositivity, } \\
n(n / N ; \%)\end{array}$ & $x^{2}$ value & $p$ value $(\mathrm{Cl} 95 \%)$ \\
\hline \multirow[t]{12}{*}{ Pregnant women $\left(N_{0}=125\right)$} & Own a cat & & & & \\
\hline & Yes & $56(44.8)$ & $28(50.0)$ & 0.058 & 0.808 \\
\hline & No & $69(55.2)$ & $36(52.1)$ & & \\
\hline & Contact with soil & & & & \\
\hline & Yes & $14(11.2)$ & $7(50.0)$ & 0.009 & 0.92 \\
\hline & No & $111(88.8)$ & $57(51.4)$ & & \\
\hline & Handle raw meat & & & & \\
\hline & Yes & $114(91.2)$ & $62(54.4)$ & 5.26 & $0.02 *$ \\
\hline & No & $11(8.8)$ & $2(18.2)$ & & \\
\hline & Eat raw meat & & & & \\
\hline & Yes & $28(22.4)$ & $14(50.0)$ & 0.02 & 0.88 \\
\hline & No & 97 (77.6) & $50(51.5)$ & & \\
\hline \multirow{12}{*}{ HIV-seropositive patients $\left(N_{0}=125\right)$} & Own a cat & & & & \\
\hline & Yes & $42(33.6)$ & $26(62.0)$ & 0.47 & 0.48 \\
\hline & No & $83(64.4)$ & $46(55.4)$ & & \\
\hline & Contact with soil & & & & \\
\hline & Yes & $64(51.2)$ & $35(54.7)$ & 0.45 & 0.49 \\
\hline & No & $61(48.8)$ & $37(60.7)$ & & \\
\hline & Handle raw meat & & & & \\
\hline & Yes & $75(60.0)$ & $40(53.3)$ & 1.39 & 0.23 \\
\hline & No & $50(40.0)$ & $32(64.0)$ & & \\
\hline & Eat raw meat & & & & \\
\hline & Yes & 111 (88.8) & $66(59.5)$ & 1.40 & 0.23 \\
\hline & No & $14(11.2)$ & $6(42.9)$ & & \\
\hline
\end{tabular}

$p$ value was calculated by Pearson's chi-square $\left(x^{2}\right)$ with confidence interval $(\mathrm{Cl})$ of $95 \%$ and 1 degree of freedom $(d f)$. $p$ values less than 0.05 were considered statistically significant and are indicated in bold fonts $\left({ }^{*}\right)$

were active infections ongoing in the pregnant women and as such the rate of congenital transmission cannot be determined.

Toxoplasmosis is the most common opportunistic infection in HIV-seropositive immune-compromised hosts, where it occurs predominantly as a reactivation of endogenous infection [35].

Until the last couple of decades, most human $T$. gondii infections were thought to be the result of contact with soil contaminated with the oocysts, since $T$. gondii oocysts can survive for years in soil [4]. Recently, many infections are thought to result from other related risk factors including cat ownership, eating raw or undercooked pork, meat and meat products, consumption of raw and unwashed vegetables $[8,9]$ and drinking from contaminated reservoir [36].

In the current study, some variables were identified as possible infection risk factors and were independently linked to a higher risk of T. gondii infection. The handling of raw meat (which includes beef and pork) among pregnant women had statistically significant association with anti-T. gondii seropositivity as a higher proportion of the pregnant women that reported handling raw meat (62, 54.4\%; $N=114$ ) had anti- $T$. gondii antibodies than the non-raw meat handlers $(2,18.2 \% ; N=11)$. Handling of raw meat was however not a significant risk factor in HIV-seropositive patients as the proportion of non-raw meat handlers with anti- $T$. gondii seropositivity (32, $64.0 \% ; N=50)$ was higher than the raw meat handlers (40, $53.3 \% ; N=75)$ although the number of the latter was higher than the former (Table 4). Toxoplasma tissue cysts contained in meat or meat-derived products have been shown to serve as important sources of infection for humans [8] and that the risk of acquiring the infection via meat sources depends on cultural and eating habits in different human populations. Cat ownership did not show any significant association with $T$. gondii seropositivity in this study. These findings are in contrast to those of 
previous studies $[8,37]$ which observed that cats play a central role in the epidemiology of $T$. gondii, constituting the only known source of environmental contamination with the infective oocyst stage. However, in support of our findings, other epidemiological studies have not shown cat ownership to be a high-risk factor for $T$. gondii infection [38] but rather exposure to the faeces from a cat that is shedding oocysts.

The seemingly high seroprevalence recorded in the children as compared to the two adult study populations may be possibly due to a variety of factors. Firstly, it is possible that the children had been exposed to risk factors that adult populations in this study had not been exposed to during their childhood. With the increase in urbanization and industrialization in many parts of Ghana, it is possible that there is an increased risk for $T$. gondii infection. The increase in urban slums is a potential high-risk point for disease transmission including $T$. gondii because of inaccessibility to clean water, unhygienic practices and inadequate sanitation. Also, the recruitment at an exclusively referral children's hospital may be a confounding factor and that results obtained may not generally be same for supposedly healthy children in the country.

\section{Conclusions}

In conclusion, the results of this study confirmed that the seroprevalence of $T$. gondii infection is high among pregnant women, children $<5$ years old and HIV-seropositive persons in Accra. The major risk factors associated with $T$. gondii seropositivity in the present study were age and contact with raw meat (among only pregnant women). A further study to investigate pre-pregnancy infections with $T$. gondii among women of childbearing age, seroconversion rate in pregnant women, rate of mother-to-child transmission and reactivated infections among HIV-seropositive persons is recommended. Such evidence will add up to existing information to improve control and management of toxoplasmosis in Ghana, especially for women of childbearing age in Ghana.

\section{Acknowledgements}

The authors acknowledge the support of the Noguchi Memorial Institute for Medical Research, Legon, and the Central Laboratory, Korle-Bu Teaching Hospital, Accra.

This research was partly supported by the Japanese Initiative for Global Research Network on Infectious Diseases (J-GRID) through the Tokyo Medical and Dental University (TMDU).

\section{Authors' contributions}

IA, TS, NO and PFA conceived and designed the study. AOS carried out data collection and performed the immunoassays. EAB performed the statistical analysis, participated in the coordination and helped to draft the manuscript. All authors read and approved the final manuscript.

\section{Competing interests}

The authors declare that they have no competing interests.

\section{Ethics approval and consent to participate}

This study was conducted in accordance with the Helsinki Declaration on Research regarding human subjects, and the study protocol was duly reviewed and approved by the Research and Ethical Review committee of the University of Ghana Medical School. Study participants aged above 18 years were recruited based on an informed written consent (that is, after the explanation of the study has been duly conducted in a language of their understanding). For children aged $<5$ years, informed consent was obtained from their parents/guardians before recruitment into the study.

Confidentiality and safety of the participants were fully assured at all times, and patient records/information was de-identified prior to analysis. Direct identifiers such as name and contact information were removed prior to analysis to protect subjects from potential risk.

\section{Author details}

${ }^{1}$ Department of Parasitology, Noguchi Memorial Institute for Medical Research, College of Health Science, University of Ghana, P. O. Box LG 581, Legon, Accra, Ghana. ${ }^{2}$ Korle-Bu Central Laboratory, Korle-Bu Teaching Hospital, P. O. Box 77, Korle-Bu, Accra, Ghana. ${ }^{3}$ Department of Environmental Parasitology, Graduate School of Medical and Dental Sciences, Tokyo Medical and Dental University, 1-5-45 Yushima, Bunkyo-ku, Tokyo 113-8519, Japan. ${ }^{4}$ School of Biological and Allied Health Sciences, College of Health Science, University of Ghana, Korle-Bu, Accra, Ghana.

Received: 5 November 2015 Accepted: 13 May 2016

Published online: 01 June 2016

\section{References}

1. Robert-Gangneux F, Darde ML. Epidemiology of and diagnostic strategies for toxoplasmosis. Clin Microbiol Revs. 2012;25:264-96.

2. Galvan-Ramirez ML, Guillén-Vargas C, Saavedra-Durán R, Islos-Rodríguez A. Analysis of Toxoplasma gondii antigens with sera from toxoplasmosis patients. Rev Soc Bras Med Trop. 1998;31:271-7.

3. Tenter AM, Heckeroth AR, Weiss LM. Toxoplasma gondii: from animals to humans. Int J Parasitol. 2000;30:1217-58.

4. Luft BJ, Conley F, Remington JS. Outbreak of central-nervous system toxoplasmosis in Western Europe and North America. Lancet. 1983;1:781-4.

5. Israelski DM, Remington JS. Toxoplasmosis in the non-AIDS immunocompromised host. Curr Clin Top Infect Dis. 1993;13:322-56.

6. Bahia-Oliveira LM, Jones JL, Azevedo-Silva J, Alves CC, Oréfice F, Addiss DG. Highly endemic waterborne toxoplasmosis in north Rio de Janeiro state, Brazil. Emer Infect Dis. 2003;9:55-62.

7. Zemene E, Yewhalaw D, Abera S, Belay T, Samuel A, Zeynudin A. Seroprevalence of Toxoplasma gondii and associated risk factors among pregnant women in Jimma town, Southwestern Ethiopia. BMC Infect Dis. 2012;12:337.

8. Abu EK, Boampong JN, Ayi I, Ghartey-Kwansah G, Afoakwah R, Nsiah P, Blay E. Infection risk factors associated with seropositivity for Toxoplasma gondii in a population-based study in the Central Region, Ghana. Epidemiol Infect. 2015;143:1904-12.

9. Portela RW, Bethony J, Costa MI, Gazzinelli A, Vitor RW, Hermeto FM, Correa-Oliveira R, Gazzinelli RT. A multi-household study reveals a positive correlation between age, severity of ocular toxoplasmosis, and levels of glycoinositolphospholipid specific immunoglobulin A. J Infect Dis. 2004; 190:175-83.

10. Montoya JG, Liensenfeld O. Toxoplasmosis. Lancet. 2004;363:1965-76.

11. Bossi P, Bricaire F. Severe acute disseminated toxoplasmosis. Lancet. 2004:364:579.

12. Berger F, Goulet V, Le Strat Y, Desenclos JC. Toxoplasmosis among pregnant women in France: risk factors and change of prevalence between 1995 and 2003. Rev Epidemiol Sante Publique. 2009;57:241-8.

13. Montoya JG, Remington JS. Management of Toxoplasma gondii infection during pregnancy. Clin Infect Dis. 2008:47:554-66.

14. Holliman RE. Congenital toxoplasmosis: prevention, screening and treatment. J Hosp Infect. 1995;30:179-90.

15. Chaisson RE, Moore RD. Prevention of opportunistic infections in the era of improved antiretroviral therapy. J AIDS Hum Retrovirol. 1997;16 Suppl 1:14-22.

16. Hunter CA, Remington JS. Immunopathogenesis of toxoplasmic encephalitis. J Infect Dis. 1994;170:1057-67.

17. Faucher B, Garcia-Meric P, Franck J, Minodier P, Francois P, Gonnet S, L'ollivier C, Piarroux R. Long term ocular outcome in congenital toxoplasmosis: a prospective cohort of treated children. J Infect. 2012;64:104-9. 
18. Niang N. Determination of sample size. Malay J Med Sc. 2003;10:84-6.

19. Ayi I, Edu SA, Apea-Kubi KA, Boamah D, Bosompem KM, Edoh D. Sero-epidemiology of toxoplasmosis amongst pregnant women in the Greater Accra Region of Ghana. Ghana Med J. 2009:43:107-14.

20. Ayeh-Kumi PF et al. Seroprevalence of toxoplasmosis among patients visiting the Korle-Bu Teaching Hospital, Accra, Ghana. Revs Infect. 2010;1:147-50.

21. Afonso E, Germain E, Poulle ML, Ruette S, Devillard S, Say L, Villena I, Aubert D, Gilot-Fromont E. Environmental determinants of spatial and temporal variations in the transmission of Toxoplasma gondii in its definitive hosts. Int J Parasitol. 2013;2:278-85.

22. Bourdhine A, Siala E, Chahed MK, Aoun K, Ben IR. Sero-epidemiologic profile of Toxoplasma gondii in Tunisia. Parasite. 2001;8:61-6.

23. Ogouyèmi-Hounto A, Agbayahoun-Chokki F, Sissinto Savi De Tove Y, Biokou Bankole B, Adinsi De Souza V, Assogba M, Kinde-Gazard D, Massougbodji A. Evaluation of a rapid diagnostic test in the diagnosis of toxoplasmosis in pregnant women in Cotonou (Bénin). Bull Soc Pathol Exot. 2014;107:85-9.

24. Hussein AH, Ali AE, Saleh MH, Nagaty IM, Rezk AY. Prevalence of Toxoplasme infection in Qualyobia governorate, Egypt. J Egypt Soc Parasitol. 2001;31:355-63.

25. Ishaku BAI, Umoh J, Lawal I, Randawa A. Seroprevalence and risk factors for Toxoplasma gondii infection among antenatal women in Zaria, Nigeria. Res J Medicine Med Sc. 2009;4:483-8.

26. Pal S, Das N, Pal D. Seroprevalence and risk factors of Toxoplasma gondii in pregnant women in Kolkata, India. J Renin-Angio-Aldo Sys. 2011;26:27-33.

27. Mwambe B, Mshana SE, Kidenya BR, Massinde AN, Mazigo HD, Michael D, Majinge C, Groß U. Seroprevalence and factors associated with Toxoplasma gondii infection among pregnant women attending antenatal care in Mwanza, Tanzania. Parasite and Vectors 2013; 6 doi: 10.1186/1756-3305-6-222.

28. Allain JP, Palmer CR, Pearson G. Epidemiological study of latent and recent infection by Toxoplasma gondii in pregnant women from a regional population in the UK. J Infect. 1998;36:189-96.

29. Robert-Gangneux F, Dupretz P, Yvenou C, Quinio D, Poulain P, Guiguen C, Gangneux JP. Clinical relevance of placenta examination for the diagnosis of congenital toxoplasmosis. Pedia Inf Dis J. 2010;29:33-8.

30. Bessieres MH, Roques C, Berrebi A, Barre V, Cazaux M, Séguéla JP. IgA antibody response during acquired and congenital toxoplasmosis. J Clin Pathol. 1992;45:605-8.

31. Uneke CJ, Duhlinska DD, Njoku MO, Ngwu BA. Seroprevalence of acquired toxoplasmosis in HIV-infected and apparently healthy individuals in Jos, Nigeria. Parasitologia. 2005;47:233-6.

32. Fan CK, Liao CW, Wu MS, Su KE, Han BC. Sero-epidemiology of Toxoplasma gondii infection among Chinese aboriginal and Han people residing in mountainous areas of northern Thailand. J Parasitol. 2003:89:1239-42.

33. Taylor MRH, Lennon B, Holland CV, Cafferkey M. Community study of Toxoplasma antibodies in urban and rural schoolchildren aged 4 to 18 years. Arch Dis Child. 1997;77:406-9.

34. Blay EA, Ghansah A, Otchere J, Koku R, Kwofie KD, Bimi L, Takashi S, Ohta N, Ayi I. Congenital toxoplasmosis and pregnancy malaria detection postpartum: effective diagnosis and its implication for efficient management of congenital infection. Parasitol Int. 2015;64:603-8.

35. Luft BJ, Remington JS. Toxoplasmic encephalitis in AIDS. Clin Infect Dis. 1992;15:211-22.

36. Cook AJC, Gilbert RE, Buffolano W, Zufferey J, Petersen E, Jenum PA, Foulon W, Semprini AE, Dunn DT. Sources of Toxoplasma infection in pregnant women. European multi-centre case-control study. BMJ. 2000;231:142-7.

37. Spalding SM, Amendoeira MR, Klein CH, Ribeiro LC. Serological screening and toxoplasmosis exposure factors among pregnant women in South of Brazil. Rev Soc Bras Med Trop. 2005;38:173-7.

38. Jones JL, Lopez B, Alvarez Mury M, Wilson M, Klein R, Luby S, Maguire JH. Toxoplasma gondii infection in rural Guatemalan children. Am J Trop Med Hyg. 2005;72:295-300.

\section{Submit your next manuscript to BioMed Central and we will help you at every step:}

- We accept pre-submission inquiries

- Our selector tool helps you to find the most relevant journal

- We provide round the clock customer support

- Convenient online submission

- Thorough peer review

- Inclusion in PubMed and all major indexing services

- Maximum visibility for your research

Submit your manuscript at www.biomedcentral.com/submit
( ) BioMed Central 